\title{
AMPUTATION IN FRACTURES OF THE TIBIA
}

\author{
J. H. Hicks, BiRmingham, ENGLAND
}

From the Birmingham Accident Hospital

Fractures of the shaft of the tibia in adults are notoriously difficult to treat and are sometimes dangerous. Poor function, non-union, skin loss and infection are the great hazards, and an awkward dilemma is posed by the fact that the treatment least likely to give one of these bad results is often the most likely to give another. Amputation, both primary and secondary, should be included among the bad results of any series since these represent complete failures. It is not uncommon to find secondary amputation referred to as though it were usually a complication of operative treatment but facts and figures are difficult to obtain. Dehne, Deffer, Hall, Brown and Johnson (1961), in a series of over 200 cases treated conservatively, had no secondary amputations. On the other hand, Holderman (1959), in a series of forty-five cases treated mostly conservatively, had three secondary amputations (6 per cent) in addition to a late primary amputation due to a tight plaster. Scott (1962), also in a predominantly conservative series, recorded two secondary amputations in 236 cases $(0.8$ per cent). The survey of Holdsworth's 99 per cent conservative series by Ellis (1956), which in other respects is comprehensive, only tabulates late primary amputations ( 2.3 per cent), but two secondary amputations $(0.5$ per cent) are mentioned in footnotes and it is by no means certain that these were all. Other authors only deal with fractures at the easy end of the range. For example, Alms (1962) includes in his series for intramedullary nailing mostly the non-comminuted and the mildest compound fractures and does not concern himself with results among the cases he rejects. Yet as Ellis (1958) pointed out the easy fractures can be expected to give good results. The bad fractures are the ones that tend to do badly.

The fact that the tragedy of amputation can occur with conservative treatment-even of simple fractures-is evident not only from Holderman's series but also from two of the cases described below. The suspicion remains, however, that operative treatment of the original fracture is likely to raise the incidence of complications. This study, therefore, of all the amputations done at the Birmingham Accident Hospital over a period of twelve years is of interest, since the policy of the Hospital is well known to be in favour of the much criticised internal fixation for the more severe cases of fractured tibia.

\section{MATERIAL}

The number of fractured shafts of tibia in adults treated in twelve years was over 1,400 . The number of legs amputated, after excluding amputations entirely due to some concomitant injury or its complications, was fifty-five. The word adult refers to persons of fifteen or over. By "shaft" is meant all cases except those in which the centre of the fracture was less than four and a half inches from the knee joint or three inches from the ankle joint. Every degree of severity of injury up to and including " traumatic amputation" had to be counted since no clear line of demarcation could be made between the almost severed limb and the gross compound fractures of the tibia with absent distal pulses, some of which have been treated with success.

\section{RESULTS}

The incidence of amputations is given year by year in Table I. Division into secondary and primary amputations is the first step towards separating those cases in which the fracture treatment could possibly have been responsible for the need to amputate from those in which 
the deciding factor was entirely the severity of the injury. Even this division is not clear-cut because of an intermediate group of eight "late primary " amputations which, in the present paper, has been included with primary amputations. In six of these the circulation was absent on admission; nevertheless an attempt had been made to preserve the limb. In one case the

TABLE I

InCidence of Amputation Due to the Fracture, or Its

COMPlications, OR ITS TREatment

\begin{tabular}{|c|c|c|c|c|}
\hline \multirow{2}{*}{ Year } & \multicolumn{2}{|c|}{ Secondary amputations } & \multicolumn{2}{|c|}{ Primary amputations } \\
\hline & Numbers & Per cent & Numbers & Per cent \\
\hline 1951 & 3) & & 3) & \\
\hline 1952 & 2 & & 7 & \\
\hline 1953 & $3\}$ & $2 \cdot 3$ & 4 & $3 \cdot 5$ \\
\hline 1954 & 2 & & 2 & \\
\hline 1955 & $4 \mathrm{~J}$ & & $5 j$ & \\
\hline 1956 & 1) & & 2) & \\
\hline 1957 & 1 & & 2 & \\
\hline 1958 & 1 & & 3 & \\
\hline 1959 & 2 & $0 \cdot 7$ & $5\}$ & $1 \cdot 7$ \\
\hline 1960 & 0 & & 1 & \\
\hline 1961 & 1 & & 1 & \\
\hline 1962 & $0\}$ & & $0 j$ & \\
\hline
\end{tabular}

TABLE II

Causes for Secondary Amputations and Their Relationship to Treatment

\begin{tabular}{|c|c|c|c|}
\hline & & & Number of cases \\
\hline \multicolumn{3}{|c|}{ Skin necrosis, sepsis, no union; with or without failed } & 9 \\
\hline Skin necrosis threatening the above seque & ence & . & 1 \\
\hline Pure sepsis & . & . & 1 \\
\hline Gas gangrene. & . & . & 1 \\
\hline Septic bone graft after initial healing & . & . & 1 \\
\hline Mechanical breakdown & . & . & 1 \\
\hline Refracture: one with recurrence of sepsis & . & . & 2 \\
\hline Large bone defect . & . & . & 1 \\
\hline Late necrosis or fibrosis of deep tissues & . & . & 3 \\
\hline
\end{tabular}

circulation disappeared during or after the primary treatment. The other case was of old osteomyelitis with fracture.

In Table II the immediate causes for the twenty secondary amputations are analysed to show their relationship to treatment.

vol. 46 B, No. 3, AUGUST 1964 


\section{DISCUSSION}

Table I shows that the secondary amputation rate (at least in recent years) is of the same order as in conservatively treated series (Ellis 1956, Holderman 1959, Scott 1962).

Table II confirms that skin necrosis, shown in Figures 1 and 2, is justifiably feared as the commonest starting point of a chain of events that leads ultimately to amputation. Primary skin loss at the time of the accident was never a factor in this series-an observation at variance with current teaching. Sepsis was rare as a primary complication; nearly always it gained access afterwards; and it was a factor influencing the decision to amputate in two-thirds of the cases. In many cases the reason for amputation was that, with non-union present or incipient, infection precluded any attempt at bone grafting. Corrosion of metal was not a cause of amputation. Osteoporosis, contractures or a painful atrophic limb, sometimes quoted as causes for amputation, accounted for three in this series. Sixteen of these twenty fractures had been compound, most of them severely, and there is, in consequence, ample to account for the amputations without necessarily incriminating the treatment. Conservative treatment is not above suspicion, at least in our hands; one of the simple fractures had been treated conservatively yet nevertheless developed skin necrosis and secondary sepsis. One hopeful feature of the series is that in recent years (with the possible exception of the last of the causes listed) cases corresponding to every group in Table II have been successfully treated and permanent healing and sound union obtained. Two such fractures are to be seen in Figures 1 and 3. It may be this improvement in treatment that has been responsible for the lowering of our amputation rate revealed in Table $I$.

Reverting to Table I, it will be observed that in 1956 the incidence of secondary amputation dropped from twenty-three per thousand to seven per thousand. This could be accounted for by a reduction in the sepsis rate or by a general decrease in the severity of fractures arriving at the hospital or by a greater readiness to do primary amputations, but there are reasons to believe that none of these explanations is the essential one. Thus Figure 3 shows that we are certainly not escaping the bad fractures nor are we making the severity of a fracture an excuse for amputation, and Table I confirms that our primary amputation rate is diminishing concurrently. The only remaining explanation is that our treatment for the complications has become more effective.

One difference between the early and the late periods of this survey was that our attitude to internal fixation had progressively hardened. Not only was the proportion of plated fractures higher in the later period but the rule that fixation should be rigid or not done at all was more constantly obeyed, and about the time of the transition between these two periods the largest plate ever to be used on the tibia was introduced. Initial treatment of the compound fracture in Figure 3, for instance, was fixation by two plates. This must not be interpreted as a recommendation for indiscriminate internal fixation. Our methods of handling both the original fractures and the complications differ from the orthodox in so many details that, until they are well understood and completely accepted, internal fixation must still be discouraged elsewhere. An earlier analysis of some of the same material, for instance, has shown that the incidence of sepsis is definitely increased by plating (Hicks 1957). Our diminishing number of amputations, however, indicates that internal fixation properly handled, far from interfering with the treatment of sepsis, can be made to facilitate it.

The most common criticism of internal fixation is that, because of sepsis, the risk of amputation is increased. In the present series, however, amputation as a result of sepsis was done in only 1 per cent ( 1.8 per cent up to 1956 and 0.4 per cent since). The assumption that all of these were the result of treatment is unjustified, just as the assumption that all amputations in a conservative school are not due to the treatment. There is some hint that conservative régimes have their own positive hazards. Thus the atrophic, painful limb is often quoted as one of the causes of late amputation whereas this factor was rare in the present series. Furthermore, the comparatively high late primary amputation rate due to 


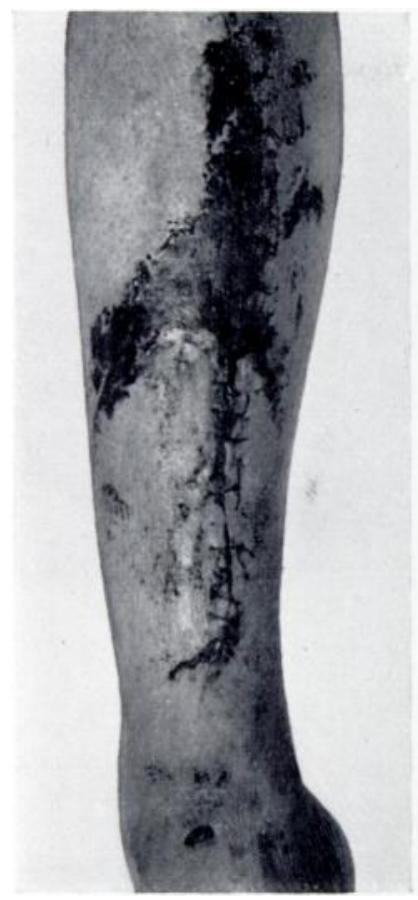

FiG. 1



FiG. 2

Figure 1-Ischaemic death of skin caused partly by stripping of the skin from its deep attachments and partly by the surgical incision. This case was brought to a successful conclusion. Figure 2-Skin defect after sloughing of necrotic skin, secondary sepsis and failed skin graft. Dead bone is present and there is no union. This leg was amputated.
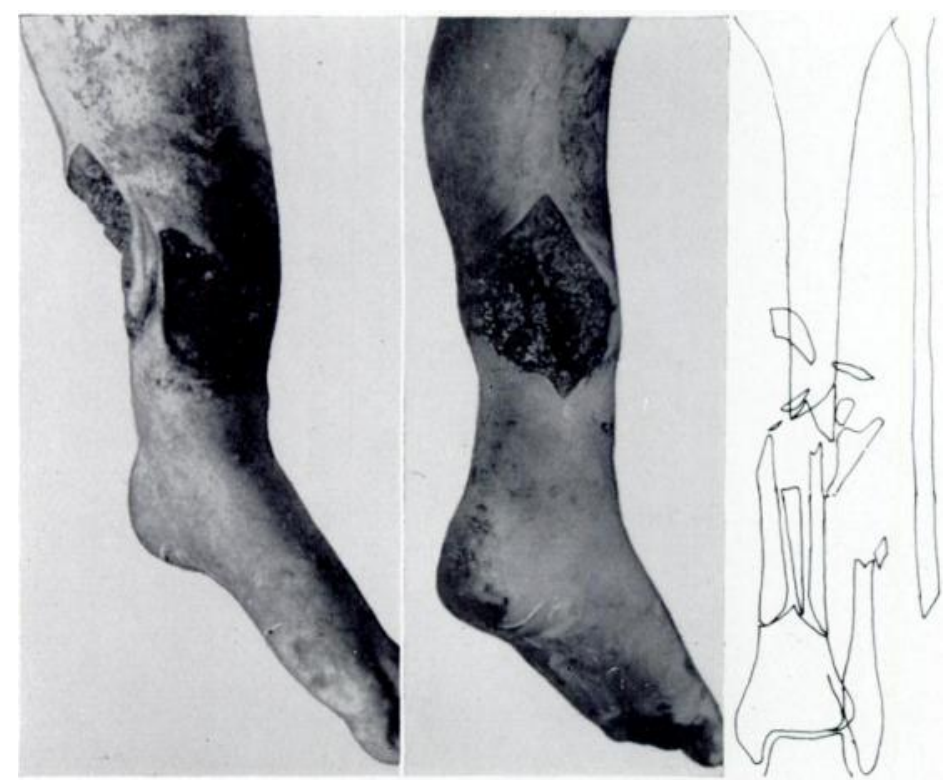

Fig. 3

The clinical appearances and radiographic tracings of a fractured tibia from the recent half of the survey. Although the foot was described as probably not viable this leg was preserved. Every complication (skin necrosis, sepsis, large bone defect and delayed union) was encountered and all were overcome. Final healing of the wounds and consolidation of the fracture were achieved after one year and the patient was back at work after fourteen months. The initial treatment was by internal fixation.

vol. 46 B, No. 3, AUGUST 1964 
circulatory failure in Ellis's series, treated mostly in plaster, suggests another hazard and the means by which it is brought about. While the subdivision into "inevitable amputations" and "iatrogenic amputations" remains impossible to assess, the above overall percentage must remain as the basis for comparison.

\section{SUMMARY}

1. The conservative school of treatment of fractures of the tibia, which bases part of its criticism of internal fixation on the ultimate risk of amputation, does not often publish its own rates of amputation.

2. Statistics from a hospital that treats one-third of closed fractured tibiae and two-thirds of compound fractures by internal fixation are therefore put up as a basis for criticism.

3. Comparisons are made with the few available statistics in the literature of conservative treatment.

4. Almost all of the causes for secondary amputation are now curable and in recent years the number of limbs being saved is increasing.

\section{REFERENCES}

ALms, M. (1962): Medullary Nailing for Fractures of the Shaft of the Tibia. Journal of Bone and Joint Surgery, 44-B, 328.

Dehne, E., Deffer, P. A., Hall, R. M., Brown, P.W., and Johnson, E. V. (1961): The Natural History of the Fractured Tibia. Surgical Clinics of North America, 41, 1,495.

Ellis, H. (1956): A Study of Some Factors Affecting Prognosis Following Tibial Shaft Fractures. Oxford: Thesis, Bodleian Library.

Ellis, H. (1958): The Speed of Healing after Fracture of the Tibial Shaft. Journal of Bone and Joint Surgery, 40-B, 42.

Hicks, J. H. (1957): The Relationship between Metal and Infection. Proceedings of the Royal Society of Medicine (Section of Orthopaedics), 50, 842.

Holderman, W. D. (1959): Results Following Conservative Treatment of Fractures of the Tibial Shaft. American Journal of Surgery, 98, 593.

Scort, J. C. (1962): In Modern Trends in Orthopaedics No. 3. Edited by J. M. P. Clark. London: Butterworths. 